\title{
THE QCD COUPLING AND PARTON DISTRIBUTIONS AT HIGH PRECISION *
}

\author{
JOHANNES BLÜMLEIN \\ Deutsches Elektronen-Synchrotron, DESY, Platanenallee 6, D-15738 Zeuthen, Germany \\ E-mail: Johannes.Bluemlein@desy.de
}

\begin{abstract}
A survey is given on the present status of the nucleon parton distributions and related precision calculations and precision measurements of the strong coupling constant $\alpha_{s}\left(M_{Z}^{2}\right)$. We also discuss the impact of these quantities on precision observables at hadron colliders.
\end{abstract}

Keywords: Deep-inelastic scattering, strong coupling constant, heavy flavors.

\section{Inside Nucleons}

The physics of the strong interactions always has been tightly connected to the study of nucleons at shorter and shorter distances. The measurement of the anomalous magnetic moments of the proton ${ }^{1}$ and neutron ${ }^{2}$ in 1933 and 1939 made clear that nucleons are no elementary particles. During the 1950ies the Hofstadter experiments $^{3}$ revealed the charge distributions inside nucleons ${ }^{4}$ at scales $Q^{2} \simeq 0.5 \cdot M_{N}^{2}$. Yet it was unknown how these distributions came about. In 1964 Murray GellMann ${ }^{5}$ proposed the quark model, to catalog the plethora of observed baryons and mesons. Independently G. Zweig suggested aces ${ }^{6}$ as the building blocks of hadrons. A direct connection to the lepton-nucleon scattering data was not made at that time.

Back in 1954 C.N. Yang and R Mill ${ }^{7}$ proposed novel bosonic field theories based on gauge invariance with respect to non-abelian groups. This development went unrelated to strong interactions for a long time. With the advent of the Stanford Linear Accelerator in 1968 the nucleon structure could be resolved at much shorter distances by the MIT-SLAC experiments 10 beyond the resonant region $W \geq 2 \mathrm{GeV}$ for values $Q^{2}$ up to $30 \mathrm{GeV}^{2}$. The remarkable finding by these experiments were that $i$ ) the structure function $\nu W_{2}\left(\nu, Q^{2}\right)$ which has been expected to depend on both kinematic variables $\nu$ and $Q^{2}$ independently, turned out to take the same values for fixed values of $x=Q^{2} /\left(2 M_{N} \nu\right)$ irrespectively of $\nu$ and $Q^{2}$ at high enough values. This phenomenon is called scaling. $i$ ) The ratio of the longitudinal structure function $W_{L}$ and $W_{2}$ turned out to be very small. Bjorken ${ }^{13}$ had predicted

*Dedicated to M. Gell-Mann on the occasion of his 80th birthday. 
scaling at asymptotic scales $Q^{2}, \nu \rightarrow \infty$ in 1969. Learning about the SLAC-MIT results R. Feynman very quickly proposed the parton mode 14 , which is equivalent to Bjorken's description but based on the observed strict microscopic correlation between $Q^{2}$ and $\nu=q \cdot p_{i}$

$$
W\left(x, Q^{2}\right)=\sum_{i} e_{i}^{2} \int_{0}^{1} d x_{i} f_{i}\left(x_{i}\right) \delta\left(\frac{q \cdot p_{i}}{M^{2}}-\frac{Q^{2}}{M^{2}}\right),
$$

where $e_{i}$ and $f_{i}$ denote the parton's charge and distribution functions. Would the parton model be unique in describing the new data? This has been challenged by other popular formalisms like vector meson dominance ${ }^{16}$. However, they failed to describe the behaviour observed for $W_{L}$, which corresponded to that of spin $1 / 2$ partons, according to the calculations by Callan and Gross 17 .

Yang-Mills theories ${ }^{7}$ became building blocks of the electro-weak Standard Mode $\frac{18}{18}$, although there renormalizibility had not been proven yet, a conditio sine qua non for a physical theory. The proof was an urgent matter and in 1971 it was achieved both for massless and spontaneously broken Yang-Mills theories, along with designing practical loop computations in this sophisticated theory in an automated way $20 \mid 26$. Quantum Chromodynamics (QCD) was proposed as the theory of the strong interactions in 1972 by Gell-Mann and Fritzsch and Leutwyler ${ }^{27 / 28}$ as a renormalized Yang-Mills field theory based on $S U(3)$ gauge interactions 29 . D. Gross, F. Wilczek ${ }^{30}$ and D. Politzer ${ }^{31}$ studied the asymptotic behaviour of color octet gluon Yang-Mills theory, cf. also 32 , and found asymptotic freedom. This is the essential ingredient, which makes it possible to perform perturbative calculations at large scales in a theory with strong interactions at low scales.

At short distances the nucleon structure functions $F_{i}\left(x, Q^{2}\right)$ obey the light-cone

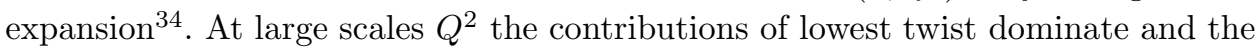
representation

$$
F_{i}\left(x, Q^{2}\right)=\sum_{j} C_{i}^{j}\left(x, Q^{2} / \mu^{2}\right) \otimes f_{j}\left(x, \mu^{2}\right)
$$

holds. Here $C_{i}^{j}\left(x, Q^{2} / \mu^{2}\right)$ denote the Wilson coefficients and $f_{j}\left(x, \mu^{2}\right)$ are the parton densities. $\mu^{2}$ is an arbitrary factorization scale and $\otimes$ denotes the Mellin convolution.

The scale behavior of the nucleon structure functions $F_{i}\left(x, Q^{2}\right)$ obey renormalization group equations, an important aspect of renormalizable Quantum Field Theories to which Murray Gell-Mann made very essential contributions very early 3 通. Transforming Eq. (2) to Mellin space one obtains the following Callan-Symanzik ${ }^{40}$ equations :

$$
\left[\mu \frac{\partial}{\partial \mu}+\beta(g) \frac{\partial}{\partial g}-2 \gamma_{\psi}(g)\right] F_{i}\left(N, Q^{2}\right)=0
$$

${ }^{a}$ It is interesting to note that different approaches to renormalization result into different mathematical structures as shown in ${ }^{38}$. Thus the method by Gell-Mann and Low $\sqrt{37}$ is in general related to a cocycle, while that by Stückelberg and Petermann $\frac{39}{2}$ relates to a group. I thank A. Petermann for pointing out Ref $[38$ to me. 


$$
\begin{aligned}
{\left[\mu \frac{\partial}{\partial \mu}+\beta(g) \frac{\partial}{\partial g}+\gamma_{\kappa}(N, \mu)-2 \gamma_{\psi}(g)\right] f_{k}\left(N, \mu^{2}\right) } & =0 \\
{\left[\mu \frac{\partial}{\partial \mu}+\beta(g) \frac{\partial}{\partial g}-\gamma_{\kappa}(N, \mu)\right] C_{j}^{k}\left(N, Q^{2} / \mu^{2}\right) } & =0,
\end{aligned}
$$

with $\beta(g)$ the QCD $\beta$-function and $\gamma_{\kappa}(N, \mu)$ the anomalous dimensions. Both functions imply the scaling violations of the structure functions $b$ With progressing time the measurement of the deep-inelastic structure functions improved considerably and after 40 years, e.g. the precision of the structure function $F_{2}^{\mathrm{em}}\left(x, Q^{2}\right)$ reached $1 \%$ over a very wide range, $\mathrm{cf} \stackrel{47}{4}$. Due to this both the precision measurement of the unpolarized parton distributions and the strong coupling constant $\alpha_{s}\left(M_{Z}^{2}\right)$ is possible from these data.

\section{Higher Order QCD Corrections to Deep-Inelastic Scattering}

On the theory side, the progress in higher order computations, likewise, has been enormous during the same period. The initial 1-loop results $30|31| 48$ are now widely improved to 3-loop order and even somewhat beyond. This is necessary to comply with the current precision of data. The status of the theory of deep-inelastic scattering is illustrated in the flowchart below. Here the dates indicate the year in which the corresponding correction to the respective quantities has been calculated.

Let me mention the most far reaching results. For the QCD $\beta$-function the $4-$ loop corrections were first computed by Vermaseren et al.$^{52}$ in 1997 . The unpolarized anomalous dimensions and massless Wilson coefficients are known to 3-loops for a series of moments 53 and in complete form $57-59$ since 2004/05. A first moment for the non-singlet+ anomalous dimensions has been computed at 4 -loops 60 in 2006 and more moments are in preparation. The heavy flavor Wilson coefficients in the region $Q^{2} / m^{2}=\rho \gg 1$, which is a good approximation in case of $F_{2}\left(x, Q^{2}\right)$ for $\rho \geq 10$, were computed for a larger number of moments 61 and in complete form for $F_{L}{ }^{[62]}$. Currently the computation of the Wilson coefficients at general values of $N$ is underway 63 .

At the level of the leading twist $(\tau=2)$ representation the light cone expansion and the QCD-improved parton model lead to the same results. In the 1980ies it was thought, that the higher order corrections need to be supplemented by different small- $x$ resummations, cf.64]65, to obtain correct results, even in the HERA kinematic region. These perturbative resummations are connected to the problem how, within their approach, perturbative and non-perturbative contributions are clearly separated - an important pre-requisite to apply perturbation theory at all.

\footnotetext{
${ }^{\mathrm{b}}$ We remind that Drell and collaborators at the end of the 1960ies ${ }^{42}$ were seeking desperately scaling in fermion-meson interactions with loop corrections but ended up with scaling violations in general.
} 


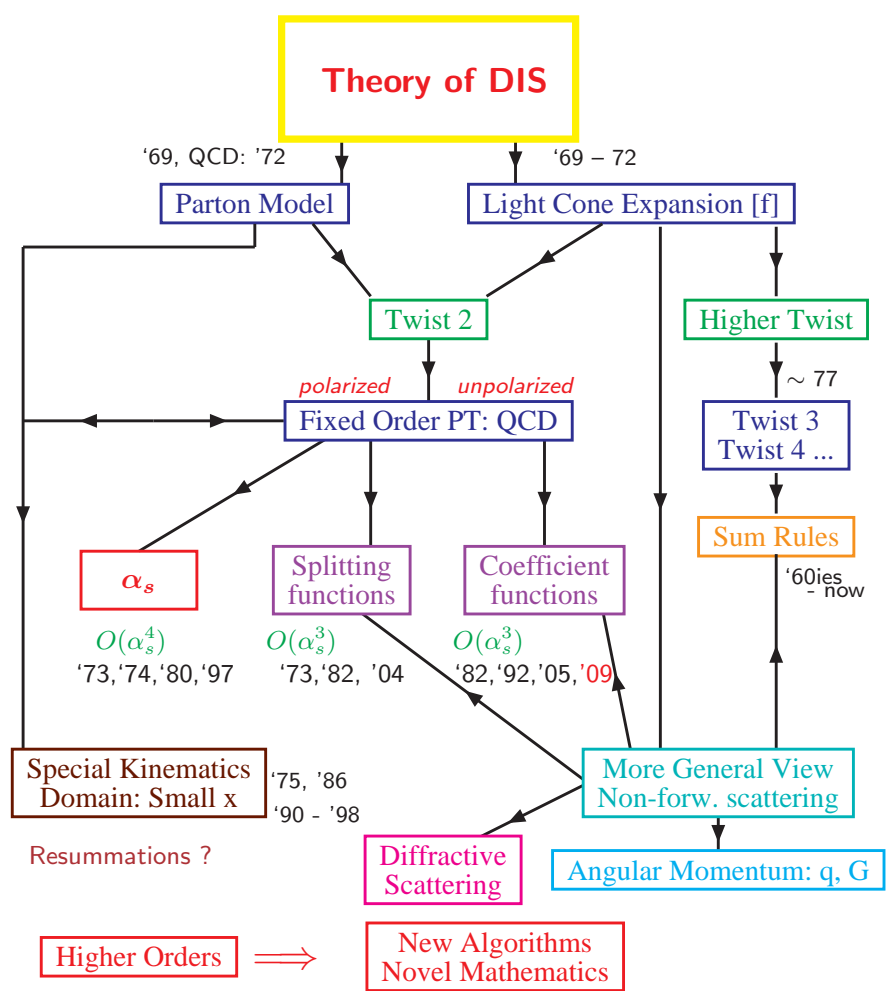

The resummation $[466$ has successfully predicted the so-called 'leading' poles of the QCD anomalous dimensions related to the poles at $N=1$ in Mellin space, at least up to $O\left(\alpha_{s}^{3}\right)$. The leading series is related to the scale-invariant limit of QCD. The corresponding resummed anomalous dimension, however, has branch cuts in the complex plane ${ }^{67}$ but no poles at all, see also $\frac{69}{}$. These singularities are much milder. Phenomenological studies $\sqrt{70}$ have shown, that subleading effects are as important as the leading ones, since they widely cancel the effect of the former. One estimates that about four complete series of these terms are needed to obtain convergence. Currently the only practical approach relies on the computation of the Wilsoncoefficients and anomalous dimensions to high enough order, which includes all the small- and large- $x$ effects automatically. In the latter case, the renormalization group even allows reliable resummations 72 .

Beyond the level of leading twist much less is known on deeply-inelastic structure functions. Most of the results obtained so far concern the 1-loop level, cf. e.g. $74[\mathrm{~g}$. Here, the corresponding partonic operator matrix elements depend on several dimensionless invariants $x_{i}$, unlike in the case of lowest twist. They cannot be measured individually in the deep-inelastic process, but require ab-initio determinations using reliable non-perturbative methods. For a series of moments, this may be possible in the future, using lattice techniques. In the polarized case a series of results has been obtained for the twist-3 contributions, cf. e.g.76, among them a series of integral relations between different structure functions ${ }^{75 / 84}$. Finally, deep-inelastic

${ }^{\mathrm{c}}$ For older references see ${ }^{75}$. 

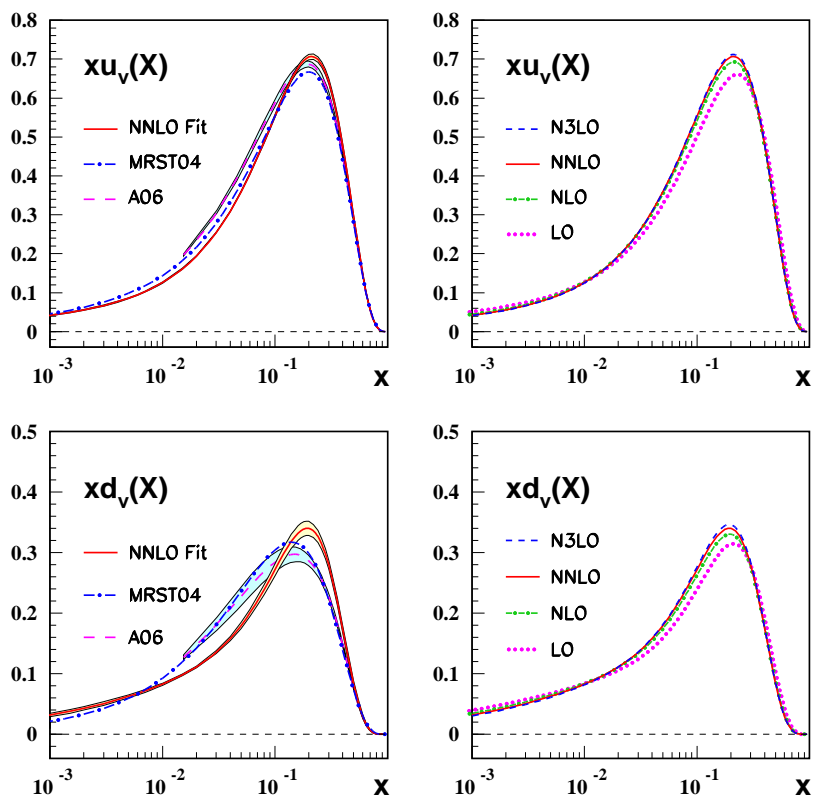

Fig. 1. Left panels: The parton densities $x u_{v}$ and $x d_{v}$ at the input scale $Q_{0}^{2}=4.0 \mathrm{GeV}^{2}$ (solid line) compared to results obtained from NNLO analyses by MRST04 (dashed-dotted line) ${ }^{90}$ and A06 (dashed line) ${ }^{91}$ The shaded areas represent the fully correlated $1 \sigma$ statistical error bands. Right panels: Comparison of the same parton densities at different orders in QCD from LO to $\mathrm{N}^{3} \mathrm{LO}$ Ref.92.

non-forward scattering has been extensively studied during the last two decades, These methods do in principle allow to measure the (quark) angular momentum of nucleons ${ }^{86}$, which is still difficult experimentally. One may apply these techniques to describe inclusive deep-inelastic diffractive scattering, referring to the light cone expansion $\frac{87}{}$ and proving that the anomalous dimensions are structurally the same as in the forward case.

\section{Precision Quark and Gluon Twist-2 Distributions}

In the following we describe recent extractions of the unpolarized twist- 2 parton densities to 3-loop accuracy and higher.

\subsection{Flavor non-singlet analysis}

The flavor non-singlet parton distributions obey scalar evolution equations and do not depend on the gluon distribution, which is more difficult to access in deepinelastic scattering and may cause some systematic uncertainty, in particular determining the strong coupling constant $\alpha_{s}\left(M_{Z}^{2}\right)$. Moreover, in the small $x$ region the QCD evolution leads to moderate changes of the the distributions, unlike in the flavor singlet and gluon-case. Due to this flavor non-singlet analyses are advantageous. 
One may apply the valence-approximation in the region $x \geq x_{0}, x_{0} \sim 0.35,0.4$ and construct a non-singlet distribution from deuteron and proton data for $x \leq x_{0}$. To describe the valence quark parton densities, the distribution $x(\bar{d}-\bar{u})\left(x, Q^{2}\right)$ has to be known, which can be measured using Drell-Yan data. Furthermore, the nonsinglet $O\left(\alpha_{s}^{2}\right)$ heavy flavor corrections are applied, which amount to about $1 \%$. The yet unknown 3-loop corrections will be even smaller. To perform a leading twist analysis, kinematic regions with higher twist contributions are cut out in a systematic study, implying the cuts of $Q^{2}>4 \mathrm{GeV}^{2}, W^{2}>12.5 \mathrm{GeV}^{2}$, cf.92|93. The results for the parton distributions $x u_{v}\left(x, Q^{2}\right)$ and $x d_{v}\left(x, Q^{2}\right)$ are illustrated in Figure 1 and are compared to other determinations. Wile in the case of the $x u_{v}$ distributions the overall agreement is good, there are still systematic differences in case of the down-valence distribution. We also illustrate the perturbative expansion from LO to $\mathrm{N}^{3} \mathrm{LO}$ reaching convergence.

Extrapolating the twist-2 QCD fit results into the region $12.5 \mathrm{GeV}^{2}>W^{2}>$ $4 \mathrm{GeV}^{2}$ the flavor non-singlet higher twist contributions can be determined empirically 94 . The inclusion of soft resummation terms for the Wilson coefficient beyond the $\mathrm{N}^{3} \mathrm{LO}$ corrections allows to extract the higher twist terms in the region $x<0.75$ in a stable way, while lower order analyses overestimate the higher twist contributions. The relative higher twist contributions in the proton and deuteron case turn out to be about of the same size.

\subsection{Combined singlet and non-singlet analysis}

In combined singlet and non-singlet analyses of the deep-inelastic world data at NNLO, cf. Refs. ${ }^{\text {95 } 99}$, one determines also the different sea-quark and gluon densities. This has always to be done together with the measurement of the QCD scale $\Lambda_{\mathrm{QCD}}$ due to strong correlations. To unfold the sea-quark densities one refers to Drell-Yan- and di-muon data as well, through which the distributions $x(\bar{d}-\bar{u})\left(x, Q^{2}\right)$ and $x s\left(x, Q^{2}\right)=x \bar{s}\left(x, Q^{2}\right)$ can be measured individually. Due to the large charmquark contribution to the deep-inelastic structure functions the description of the heavy flavor contributions is required at the same level of accuracy as for the light partons. Currently it is available to $O\left(\alpha_{s}^{2}\right)^{\sqrt{100}}$ and the $O\left(\alpha_{s}^{3}\right)$ corrections are underway $6163 \mid 102$. To obtain an accurate interpolation between low and higher scales $Q^{2}$, the so-called BMSN-interpolation is recommended ${ }^{103 \mid 104}$. Nowhere in the kinematic region of HERA heavy flavor logs become large to be resummed 105 , i.e. in a very wide kinematic region even the charm quarks cannot be viewed massless. Yet, one may define heavy flavor parton densities in terms of technical quantities $61 \mid 103$ to some extent d to evaluate other observables. Here one has always to check to which extent this approximation holds.

The present analyses have lead to very precise parton distributions. The NNLO parton distributions determined in Refs $\frac{95 \mid 97}{10}$ do widely agree within the measured

d At 3-loop order graphs exist with both charm and bottom-quark lines in the operator matrix elements. They do not fall under the paradigm of single parton distributions, despite being universal. 

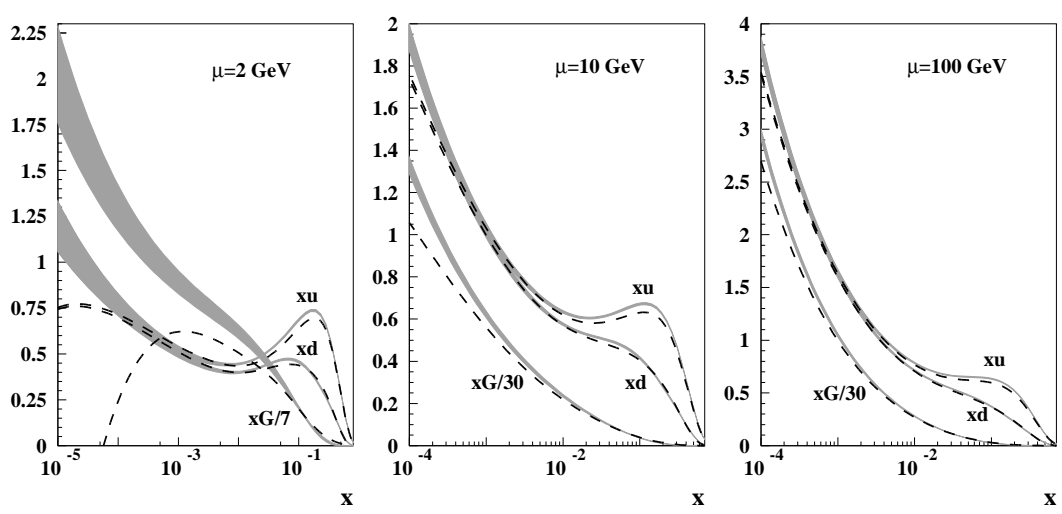

Fig. 2. The light parton densities $x u, x d$ and $x G$ at the scales $\mu^{2}=4,100,10000 \mathrm{GeV}^{2}$. The bands denote the parton distributions with $1 \sigma$ uncertainty of ABKM0995. The dashed lines correspond to MSTW08 99 ; from Ref 95 .

region, with very slight differences in the $x(u+\bar{u})$ and $x(d+\bar{d})$ distributions. In Figure 2 we compare the results of the NNLO fits of Refs ${ }^{95 \mid 99}$ for the light partons. At low scales $\mu^{2}$ the sea-quark and gluon distributions 99 take lower values than those in Ref. ${ }^{95}$, and the NNLO gluon distribution even tends to negative values yielding the largest difference.

Through the evolution the densities get closer. This, however, is partly due to the large value of $\alpha_{s}\left(M_{Z}^{2}\right)$, Eq. (14), which leads to a relative acceleration of the evolution compared to $\frac{95}{\text {. }}$. At scales larger than $Q^{2} \sim 10^{4} \mathrm{GeV}^{2}$, accessible at the LHC, this will lead to a further growing gluon density of 199 compared to $\frac{95}{\text {. The }}$ precision observables at the LHC will help to constrain the parton distributions further.

\section{The Strong Coupling Constant}

The QCD parameter $\Lambda_{\mathrm{QCD}}$, or $\alpha_{s}\left(M_{Z}^{2}\right)$, is determined in QCD fits together with the non-perturbative input densities for the different partons at a starting scale $Q_{0}^{2}$. There are tight correlations between the value of $\alpha_{s}\left(M_{Z}^{2}\right)$ and some parameters of the parton densities. An important example is the normalization of the gluon density, cf. ${ }^{95 / 106}$. In the non-singlet analysis ${ }^{92}$ we obtained at NNLO, cf. also ${ }^{107}$,

$$
\Lambda_{\mathrm{QCD}}^{N_{f}=4}=226 \pm 25 \mathrm{MeV}
$$

and at $\mathrm{N}^{3} \mathrm{LO}$, assigning to the yet unknown 4-loop anomalous dimension a $\pm 100 \%$ error,

$$
\Lambda_{\mathrm{QCD}}^{N_{f}=4}=234 \pm 26 \mathrm{MeV}
$$


Usually the QCD parameter is expressed in terms of $\alpha_{s}\left(M_{Z}^{2}\right)$. In the following we compare the results of recent $\mathrm{NNLO}$ and $\mathrm{N}^{3} \mathrm{LO}$ analyses for the deep-inelastic world data obtained by different groups :

$$
\begin{aligned}
& \alpha_{s}\left(M_{Z}^{2}\right)=0.1134_{-0.0021}^{+0.0019} \text { NNLO } \\
& \alpha_{s}\left(M_{Z}^{2}\right)=0.1141_{-0.0022}^{+0.0020} \mathrm{~N}^{3} \mathrm{LO} \\
& \alpha_{s}\left(M_{Z}^{2}\right)=0.1135 \pm 0.0014 \quad \text { NNLO, FFS } \\
& \alpha_{s}\left(M_{Z}^{2}\right)=0.1129 \pm 0.0014 \quad \text { NNLO, BSMN [95] } \\
& \alpha_{s}\left(M_{Z}^{2}\right)=0.1124 \pm 0.0020 \quad \text { NNLO, dyn. approach } 97 \\
& \alpha_{s}\left(M_{Z}^{2}\right)=0.1158 \pm 0.0035 \quad \text { NNLO, stand. approach 97. } \\
& \alpha_{s}\left(M_{Z}^{2}\right)=0.1171 \pm 0.0014 \quad \text { NNLO [108 }
\end{aligned}
$$

More recent unpolarized NNLO fits, including the combined HERA data ${ }^{47}$, yield

$$
\begin{array}{ll}
\alpha_{s}\left(M_{Z}^{2}\right)=0.1147 \pm 0.0012 \mathrm{NNLO} & {[96} \\
\alpha_{s}\left(M_{Z}^{2}\right)=0.1145 \pm 0.0042 \mathrm{NNLO}, \text { preliminary } & {[109}
\end{array}
$$

Note that the values (819) are independent of the gluon distribution. The combined singlet non-singlet analysis, based on rather different data, and being sensitive to both the sea-quark and gluon densities, yield the very similar values (1011). This analysis has been performed with a different code than used in 92 .

The above values are located below the present weighted average of $\alpha_{s}\left(M_{Z}^{2}\right)$ measurements 110 of

$$
\alpha_{s}\left(M_{Z}^{2}\right)=0.1184 \pm 0.0007,
$$

cf. Figure 3. The error given in (17) cannot include the yet unknown relative systematics between the different classes of the same type of measurement.

We would like to mention that recent determinations of $\alpha_{s}\left(M_{Z}^{2}\right)$ using event shape moments for high energy $e^{+} e^{-}$annihilation data from PETRA and LEP including power corrections the following values were obtained :

$$
\begin{array}{ll}
\alpha_{s}\left(M_{Z}^{2}\right)=0.1135 \pm 0.0002(\exp ) \pm 0.005\left(\Omega_{1}\right) \pm 0.0009 \text { (pert) NNLO [11] } \\
\alpha_{s}\left(M_{Z}^{2}\right)=0.1153 \pm 0.0017(\exp ) \pm 0.0023(\text { th }) & \text { NNLO [112]. }
\end{array}
$$

Also these measurements of $\alpha_{s}\left(M_{Z}^{2}\right)$ yield low values. They show that the results obtained analyzing deep-inelastic data do not form a special case. Also in deepinelastic scattering off polarized targets $\alpha_{s}\left(M_{Z}^{2}\right)$, at NLO, has been measured, however with larger errors, see $106\left[113\left[114\right.\right.$. The present error on $\alpha_{s}\left(M_{Z}^{2}\right)$ at NNLO of $\sim 0.0012$ is at the margin of the present theory and systematics errors. Different known theoretical uncertainties, cf. also Eqs. (811), are of the order of 0.0007 , the quoted $1 \sigma$ error of the world average. The systematics of the different extractions of $\alpha_{s}\left(M_{Z}^{2}\right)$ has to be understood in even more detail in the future. The current values of $\alpha_{s}\left(M_{Z}^{2}\right)$ obtained from precision deep-inelastic scattering data disfavor the 


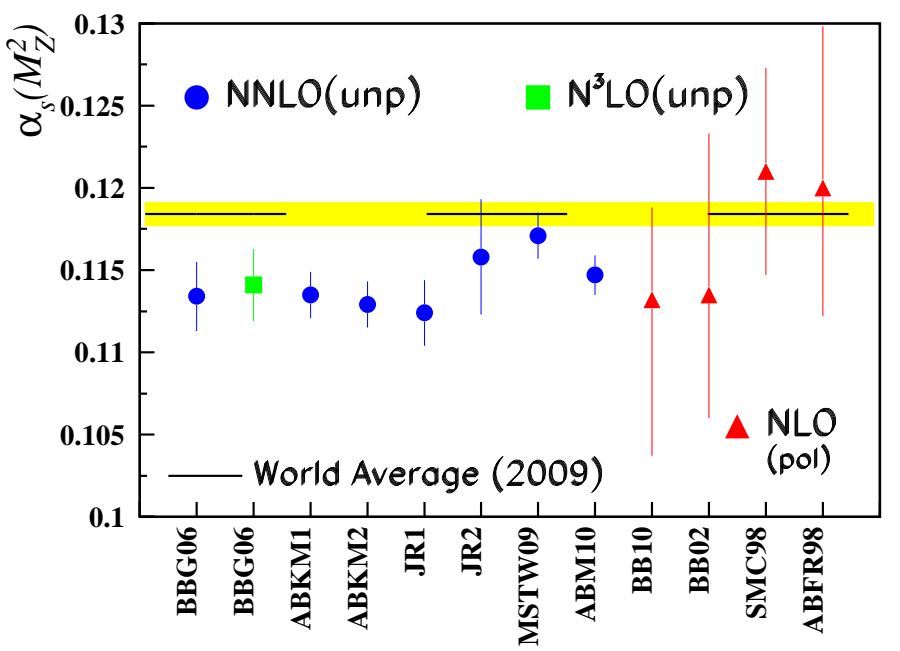

Fig. 3. The strong coupling constant $\alpha_{s}\left(M_{Z}^{2}\right)$ from different DIS measurements, at NNLO and $\mathrm{N}^{3}$ LO, Eqs. 8 15, and at NLO in the polarized case, cf.106114. The yellow band describes the weighted average of a wide range of $\alpha_{s}\left(M_{Z}\right)$ measurements 110 ; Ref [106].

unification of forces, even in the supersymmetric extension of the Standard Model. However, the picture may change soon, with new findings at the LHC.

\section{Main Inclusive Cross Sections at Hadron Colliders}

The accuracy for the parton distribution functions reached can now be applied to derive precision predictions for inclusive hadronic observables, such as the Drell-Yan cross section, the $W^{ \pm}, Z$-boson, the $t \bar{t}$ - and Higgs-boson production cross sections at NNLO. Detailed analyses have been given in ${ }^{95}$ [99. For all these quantities at least these corrections are necessary. The Drell-Yan cross section and the $W^{ \pm}, Z$-boson production cross sections are, furthermore, used as 'standard candle' processes to measure the collider luminosity. They have therefore to be known as precisely as possible. As an example we show in Figure 4 predictions on the inclusive Higgs boson production at hadron colliders. Within the present accuracy of the parton distribution functions the predictions ${ }^{95 / 99}$ still show some differences, which are likely related to the different gluon distributions at low scale and the values of $\alpha_{s}\left(M_{Z}^{2}\right)$. The predictions agree for $s \sim M_{Z}$ but differ for higher mass scales.

\section{Higher Loop Integrals and Mathematics}

The computation of higher order loop integrals is still a difficult task, even in the massless case or in the presence of a single mass scale. At three-loop (and higher) orders this both applies to the zero- and single-scale problems calculated at present. At the one hand, the results expected have a rather simple structure. On the other hand, a growing multitude of diagrams which contain more and more difficult struc- 


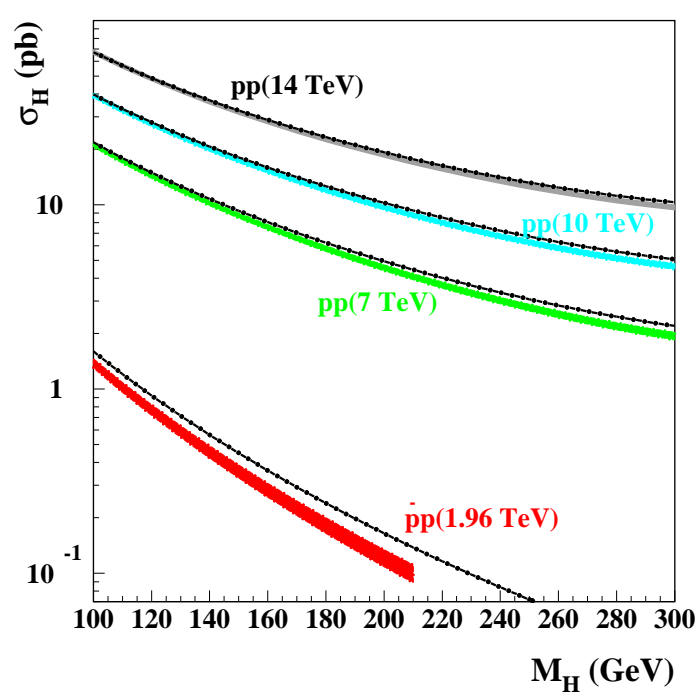

Fig. 4. Inclusive Higgs boson production cross section at TEVATRON and the LHC. The bands denote the parton distribution uncertainty of ABKM0 $\sqrt{95}$; the lines correspond to MSTW0899; from Ref $\frac{95}{}$

tures have to be computed. Obviously, an enormous part of intermediary results simply cancels. It is, however, difficult to let cancel these contributions at a rather early stage of the computation, or to even widely avoid that they occur from the very beginning. This is one of the central problems of all present calculations. Gauss' theorem $\frac{115}{}$ allows to express Feynman diagrams to a set of master integrals, which finally have to be computed for zero scale quantities. Also in case of single scale quantities, e.g. given by a Mellin variable $N$, one may obtain similar recursions. This method, simplifying the calculation, may lead to a large number of terms being of higher complexity than those finally appearing in the results. This property seems to be in common with different other approaches, which in the first place appear to simplify the calculation technically, like Mellin-Barnes 118 integrals or multinomial expansion, since they lead to rather elementary Feynman parameter integrals. Another approach, cf. e.g.631021121122 , consists in evaluating the individual Feynman parameter integrals without applying intermediary simplifying methods, i.e. mapping them directly to the analytic mathematical structure they represent. This method is more demanding but will finally lead us to a deeper understanding of the objects we deal with. For single scale single integrals integrals and one mass, the 2-loop integrals and simpler 3-loop integrals can be represented in terms of generalized hypergeometric functions and extensions thereof, like Appell functions cf.123. An important issue in integration is a clear definition of the target space and the knowledge of the relations of its elements. For the Feynman parameter integrals discussed above, shuffle- and Hopf-algebras ${ }^{125}$ play a central role, along with 
Poincaré iterated integrals over specific alphabets. Feynman integrals are almost always periods 129 . In this context, the simplest structures are multiple zeta values, cf. $\frac{131}{13}$, nested harmonic sums ${ }^{132}$, harmonic polylogarithms ${ }^{134}$, and generalized harmonic sums 135137. Feynman parameter integrals will coin new classes of higher transcendental functions going to higher and higher order, which have to be studied to perform future precision calculations in an efficient way. Their evaluation is intimately connected to modern summation technologies, like SIGMA 138 , and efficient algorithms to establish and solve the associated recurrences of both large order and degree ${ }^{63 \mid 139}$. All this will require high performance computer algebra written using highly efficient languages like FORN ${ }^{140}$, nearly 50 years after SCHOONSCHIP was introduced ${ }^{26}$, and the investment of many CPU years, however, at an even more involved level than considered today. Structures of Feynman parameter integrals, e.g. on the level of multiple zeta values, form an interesting recent field in mathematics, cf. $\frac{122] 141142}{}$, which is also related to irrationality proofs of the basis elements spanning these quantities. Here we face a new era of a tight symbiosis between theoretical physics and modern mathematics, which is regarded to be very essential.

\section{Conclusions}

Quantum Chromodynamics was a great discovery. With it Murray Gell-Mann completed the revolution of the strong interactions started in the early 1960ies with the introduction of the quarks. During the last 37 years computations grew to a precision of $O(1 \%)$ for inclusive quantities, which are described at 3-loop, and partly at 4-loop, level, moving the frontiers of Quantum Field Theory to breathtaking new horizons. The running of the strong coupling constant is understood in great detail, despite in different classes of analyses still values of $\alpha_{s}\left(M_{Z}^{2}\right)$ are found which differ by experimental and theoretic systematic effects, being partly yet unknown. To determine $\alpha_{s}\left(M_{Z}^{2}\right)$ at the level of its present statistical accuracy of $\sim 1 \%$ further studies and even higher order calculations are required for some of the processes. The QCD improved quark-gluon parton model works impressively well at short distances - a clear triumph of Quantum Chromodynamics and proof that quarks and gluons, although being confined, are basic building blocks of matter. Without them the Standard Model would suffer from anomalies ${ }^{143}$ and not form a Quantum Field Theory.

During the last two decades the methods of lattice QCD steadily improved, in particular concerning the systematic errors involved. We therefore expect precision computations both on $\Lambda_{\mathrm{QCD}}$ and a series of moments of unpolarized and polarized parton densities in the near future. The results of these calculations ab initio can then be compared to the precision extractions discussed based on precision experimental data and higher order perturbative calculations. A final question concerns the unification of the three forces of the Standard Model, given what we know at low scales at present. Future discoveries, perhaps at the LHC, will lead to a clarification here. 
Acknowledgment. I would like to thank the organizers of the conference for invitation. In a conversation with M. Gell-Mann it turned out that we have a second common interest: the physics of the direction of time. Everywhere in physics it runs forward, for all we know.

\section{References}

1. R. Frisch and O. Stern, Z. Phys. 85, p. 4 (1933).

2. L. W. Alvarez and F. Bloch, Phys. Rev. 57, 111 (1940).

3. R. Hofstadter Electron scattering and nuclear and nucleon structure. A collection of reprints with an introduction, (New York, Benjamin, 1963), $690 \mathrm{p}$.

4. D. N. Olson, H. F. Schopper and R. R. Wilson, Phys. Rev. Lett. 6, 286 (1961).

5. M. Gell-Mann, Phys. Lett. 8, 214 (1964).

6. G. Zweig An $S U(3)$ model for the strong interaction symmetry and its breaking, CERN-TH-401, 412 (1964).

7. C.-N. Yang and R. L. Mills, Phys. Rev. 96, 191 (1954).

8. W. K. H. Panofsky Low $q^{2}$ electrodynamics, elastic and inelastic electron (and muon) scattering, Proc. 14th International Conference on High-Energy Physics, Vienna, 1968, J. Prentki and J. Steinberger, eds., (CERN, Geneva, 1968), pp. 23.

9. R. E. Taylor Inelastic electron - proton scattering in the deep continuum region, Proc. 4th International Symposium on Electron and Photon Interactions at High Energies, Liverpool, 1969, (Daresbury Laboratory, 1969), eds. D.W. Braben and R.E. Rand, pp. 251.

10. H. W. Kendall, Rev. Mod. Phys. 63, 597 (1991).

11. R. E. Taylor, Rev. Mod. Phys. 63, 573 (1991).

12. J. I. Friedman, Rev. Mod. Phys. 63, 615 (1991).

13. J. D. Bjorken, Phys. Rev. 1791547 (1969).

14. R. P. Feynman The behavior of hadron collisions at extreme energies, Proc. of 3rd International Conference on High Energy Collisions, Stony Brook, 1969, C.N. Yang, J.A. Cole, M. Good, R. Hwa, and J. Lee-Franzini, eds., (Gordon and Breach, New York, 1970), pp. 237.

15. R. P. Feynman Photon-hadron interactions, (Benjamin Press, Reading, 1972), 282 p.

16. J. Sakurai Vector-Meson dominance - present status and future prospects, Proc. 4th International Symposium on Electron and Photon Interactions at High Energies, Liverpool, 1969, (Daresbury Laboratory, 1969), eds. D.W. Braben and R.E. Rand, pp. 91.

17. C. G. Callan and D. J. Gross, Phys. Rev. Lett. 22, 156 (1969).

18. S. L. Glashow, Nucl. Phys. 22, 579 (1961).

19. S. Weinberg, Phys. Rev. Lett. 19, 1264 (1967).

20. G. 't Hooft, Nucl. Phys. B33, 173 (1971).

21. G. 't Hooft and M. J. G. Veltman, Nucl. Phys. B44, 189 (1972).

22. G. 't Hooft and M. J. G. Veltman, Nucl. Phys. B50, 318 (1972).

23. G. 't Hooft, Nucl. Phys. B61, 455 (1973).

24. G. 't Hooft and M. J. G. Veltman Diagrammar, CERN Yellow Report 73-9 (1973).

25. G. 't Hooft and M. J. G. Veltman, Nucl. Phys. B153, 365 (1979).

26. M. J. G. Veltman SCHOONSCHIP, version 1, Dec. 1963.

27. H. Fritzsch and M. Gell-Mann Current algebra: Quarks and what else?, Proceedings of 16th International Conference on High-Energy Physics, Batavia, Illinois, 6-13 Sep Vol. 2, J.D. Jackson, A. Roberts, R. Donaldson, eds., pp. 135 (1972), hep-ph/0208010.

28. H. Fritzsch, M. Gell-Mann and H. Leutwyler, Phys. Lett. B47, 365 (1973).

29. Y. Nambu A Systematics Of Hadrons In Subnuclear Physics, in: Preludes in Theo- 
retical Physics, eds. A. De-Shalit, H. Fehsbach and L. van Hove (North-Holland, Amsterdam, 1966), pp. 133.

30. D. J. Gross and F. Wilczek, Phys. Rev. Lett. 30, 1343 (1973).

31. H. D. Politzer, Phys. Rev. Lett. 30, 1346 (1973).

32. I. B. Khriplovich, Yad. Fiz. 10, 409 (1969).

33. G. t'Hooft (1972), unpublished.

34. K. G. Wilson, Phys. Rev. 179, 1499 (1969).

35. R. A. Brandt and G. Preparata, Nucl. Phys. B27, 541 (1972).

36. Y. Frishman, Annals Phys. 66, 373 (1971).

37. M. Gell-Mann and F. E. Low, Phys. Rev. 95, 1300 (1954).

38. R. Brunetti, M. Duetsch and K. Fredenhagen (2009), arXiv:0901.2038 [math-ph].

39. E. C. G. Stückelberg and A. Petermann, Helv. Phys. Acta 24, 317 (1951).

40. C. G. Callan, Phys. Rev. D2, 1541 (1970).

41. K. Symanzik, Commun. Math. Phys. 18, 227 (1970).

42. S. D. Drell, D. J. Levy and T.-M. Yan, Phys. Rev. 187, 2159 (1969).

43. S. D. Drell, D. J. Levy and T.-M. Yan, Phys. Rev. D1, 1617 (1970).

44. S. D. Drell, D. J. Levy and T.-M. Yan, Phys. Rev. D1, 1035 (1970).

45. T.-M. Yan and S. D. Drell, Phys. Rev. D1, 2402 (1970).

46. S. D. Drell and T.-M. Yan, Ann. Phys. 66, 578 (1971).

47. F. Aaron et al., JHEP 1001, p. 109 (2010).

48. D. J. Gross and F. Wilczek Phys. Rev. D8, 3633 (1973).

49. D. J. Gross and F. Wilczek Phys. Rev. D9, 980 (1974).

50. H. Georgi and H. D. Politzer, Phys. Rev. D9, 416 (1974).

51. W. Furmanski and R. Petronzio, Z. Phys. C11, 293 (1982), and references therein.

52. J. A. M. Vermaseren, S. A. Larin and T. van Ritbergen, Phys. Lett. B405, 327 (1997).

53. S. A. Larin, T. van Ritbergen and J. A. M. Vermaseren, Nucl. Phys. B427, 41 (1994).

54. S. A. Larin, P. Nogueira, T. van Ritbergen and J. A. M. Vermaseren, Nucl. Phys. B492, 338 (1997).

55. A. Retey and J. A. M. Vermaseren, Nucl. Phys. B604, 281 (2001).

56. J. Blümlein and J. A. M. Vermaseren, Phys. Lett. B606, 130 (2005).

57. S. Moch, J. A. M. Vermaseren and A. Vogt, Nucl. Phys. B646, 181 (2002).

58. A. Vogt, S. Moch and J. A. M. Vermaseren, Nucl. Phys. B691, 129 (2004).

59. J. A. M. Vermaseren, A. Vogt and S. Moch, Nucl. Phys. B724, 3 (2005).

60. P. A. Baikov and K. G. Chetyrkin, Nucl. Phys. Proc. Suppl. 160, 76 (2006).

61. I. Bierenbaum, J. Blümlein and S. Klein, Nucl. Phys. B820, 417 (2009).

62. J. Blümlein, A. De Freitas, W. L. van Neerven and S. Klein, Nucl. Phys. B755, 272 (2006).

63. J. Ablinger, I. Bierenbaum, J. Blümlein et al. (2010), arXiv:1007.0375, Nucl. Phys. (Proc. Suppl.) B, in print.

64. V. S. Fadin, E. A. Kuraev and L. N. Lipatov, Phys. Lett. B60, 50 (1975).

65. L. V. Gribov, E. M. Levin and M. G. Ryskin, Nucl. Phys. B188, 555 (1981).

66. V. S. Fadin and L. N. Lipatov, Phys. Lett. B429, 127 (1998).

67. R. Ellis, F. Hautmann and B. Webber, Phys. Lett. B348, 582 (1995).

68. J. Blümlein (1995), hep-ph/9506446.

69. J. Blümlein and W. L. van Neerven, Phys. Lett. B450, 412 (1999).

70. J. Blümlein and A. Vogt Phys. Lett. B370, 149 (1996), hep-ph/9510410 Acta Phys. Polon. B27, 1309 (1996), hep-ph/9603450; Phys. Lett. B386, 350 (1996), hep-ph/9606254 Phys. Rev. D58, 014020 (1998), hep-ph/9712546.

71. J. Blümlein, V. Ravindran, W. L. van Neerven and A. Vogt (1998), hep-ph/9806368 
72. G. F. Sterman, Partons, factorization and resummation, TASI lectures, hep-ph/9606312, (1995).

73. E. Laenen, G. Stavenga and C. D. White, JHEP 03, p. 054 (2009).

74. V. Braun, A. Manashov and J. Rohrwild, Nucl. Phys. B826, 235 (2010).

75. J. Blümlein and A. Tkabladze, Nucl. Phys. B553, 427 (1999).

76. J. Kodaira, Y. Yasui and T. Uematsu, Phys. Lett. B344, 348 (1995).

77. B. Geyer, D. Müller and D. Robaschik (1996), hep-ph/9611452

78. J. Kodaira and K. Tanaka, Nucl. Phys. (Proc. Suppl.) B 86, 134 (2000).

79. X.-D. Ji, W. Lu, J. Osborne and X.-T. Song, Phys. Rev. D62, 094016 (2000).

80. A. V. Belitsky, X.-D. Ji, W. Lu and J. Osborne, Phys. Rev. D63, 094012 (2001).

81. V. M. Braun, G. Korchemsky and A. Manashov, Nucl. Phys. B597, 370 (2001).

82. V. M. Braun, G. Korchemsky and A. Manashov, Nucl. Phys. B603, 69 (2001).

83. V. Braun, A. Manashov and B. Pirnay, Phys. Rev. D80, 114002 (2009).

84. J. Blümlein and N. Kochelev, Nucl. Phys. B498, 285 (1997).

85. A. Belitsky and A. Radyushkin, Phys. Rep. 418, 1 (2005).

86. X.-D. Ji, Phys. Rev. Lett. 78, 610 (1997).

87. J. Blümlein, B. Geyer and D. Robaschik, Nucl. Phys. B560, 283 (1999).

88. J. Blümlein and D. Robaschik, Phys. Lett. B517, 222 (2001).

89. J. Blümlein and D. Robaschik, Phys. Rev. D65, 096002 (2002).

90. A. Martin, R. Roberts, W. Stirling and R. Thorne, Phys. Lett. B604, 61 (2004).

91. S. Alekhin, K. Melnikov and F. Petriello, Phys. Rev. D74, 054033 (2006).

92. J. Blümlein, H. Böttcher and A. Guffanti, Nucl. Phys. B774, 182 (2007).

93. J. Blümlein, H. Böttcher and A. Guffanti, Nucl. Phys. Proc. Suppl. 135, 152 (2004).

94. J. Blümlein and H. Böttcher, Phys. Lett. B662, 336 (2008).

95. S. Alekhin, J. Blümlein, S. Klein and S. Moch, Phys. Rev. D81, 014032 (2009).

96. S. Alekhin, J. Blümlein and S. Moch (2010), arXiv:1007.3657.

97. P. Jimenez-Delgado and E. Reya, Phys. Rev. D79, 074023 (2009).

98. P. Jimenez-Delgado and E. Reya, Phys. Rev. D80, 114011 (2009).

99. A. D. Martin, W. J. Stirling, R. S. Thorne and G. Watt, Eur. Phys. J. C63, 189 (2009).

100. E. Laenen, S. Riemersma, J. Smith and W. L. van Neerven, Nucl. Phys. B392, 162 (1993); B392, 229 (1993).

101. S. Riemersma, J. Smith and W. L. van Neerven, Phys. Lett. B347, 143 (1995).

102. I. Bierenbaum, J. Blümlein, S. Klein and C. Schneider, Nucl. Phys. B803, 1 (2008).

103. M. Buza, Y. Matiounine, J. Smith and W. L. van Neerven, Eur. Phys. J. C1, 301 (1998).

104. I. Bierenbaum, J. Blümlein and S. Klein, Phys. Lett. B672, 401 (2009).

105. M. Glück, E. Reya and M. Stratmann, Nucl. Phys. B422, 37 (1994).

106. J. Blümlein and H. Böttcher, arXiv:1005.3113, (2010).

107. M. Glück, E. Reya and C. Schuck, Nucl. Phys. B754, 178 (2006).

108. A. D. Martin, W. J. Stirling, R. S. Thorne and G. Watt, Eur. Phys. J. C 64653 (2009).

109. H1 and ZEUS collab. (V. Radescu et al.), Combined H1 and ZEUS Fits Using Low Energy Data, talk, DIS 2010, Florence, April 2010.

110. S. Bethke, Eur. Phys. J. C64, 689 (2009).

111. R. Abbate, M. Fickinger, A. H. Hoang, V. Mateu and I. W. Stewart, arXiv:1006.3080 [hep-ph].

112. T. Gehrmann, M. Jaquier and G. Luisoni, Eur. Phys. J. C 6757 (2010).

113. J. Blümlein and H. Böttcher, arXiv:1007.2784, (2010).

114. J. Blümlein and H. Böttcher, Nucl. Phys. B636, 225 (2002). 
115. J. Lagrange Nouvelles recherches sur la nature et la propagation du son, Miscellanea Taurinensis, t. II, 1760-61; Oeuvres t. I, p. 263.

116. K. G. Chetyrkin, A. L. Kataev and F. V. Tkachov, Nucl. Phys. B174, 345 (1980).

117. S. Laporta, Int. J. Mod. Phys. A15, 5087 (2000).

118. E. Barnes, Proc. Lond. Math. Soc. 6, 141 (1908).

119. E. Barnes, Quart. J. Math. 41, 136 (1910).

120. H. Mellin, Math. Ann. 68, 305 (1910).

121. I. Bierenbaum, J. Blümlein and S. Klein, Nucl. Phys. B780, 40 (2007).

122. F. Brown, Commun. Math. Phys. 287, 925 (2009).

123. W. Bailey Generalized Hypergeometric Series, (Cambridge University Press, Cambridge, 1935), $108 \mathrm{p}$.

124. L. Slater Generalized Hypergeometric Functions, (Cambridge University Press, Cambridge, 1966), $273 \mathrm{p}$.

125. D. Kreimer, Adv. Theor. Math. Phys. 2, 303 (1998).

126. A. Connes and D. Kreimer, Commun. Math. Phys. 199, 203 (1998).

127. D. J. Broadhurst and D. Kreimer, J. Symb. Comput. 27, p. 581 (1999).

128. S. Weinzierl, Eur. Phys. J. C33, s871 (2004).

129. C. Bogner and S. Weinzierl, J. Math. Phys. 50, p. 042302 (2009).

130. C. Bogner and S. Weinzierl, Int. J. Mod. Phys. A25, 2585 (2010).

131. J. Blümlein, D. Broadhurst and J. Vermaseren, Comput. Phys. Commun. 181, 582 (2010), and references therein.

132. J. Blümlein and S. Kurth, Phys. Rev. D60, p. 014018 (1999).

133. J. A. M. Vermaseren, Int. J. Mod. Phys. A14, 2037 (1999).

134. E. Remiddi and J. A. M. Vermaseren, Int. J. Mod. Phys. A15, 725 (2000).

135. A. Goncharov, Math. Res. Lett. 5 (1998) 497.

136. S. Moch, P. Uwer and S. Weinzierl, J. Math. Phys. 43, 3363 (2002).

137. J. Ablinger, J. Blümlein and C. Schneider (2010), in preparation.

138. C. Schneider J. Symbolic Comput. 43, 611 (2008), arXiv:0808.2543, Ann. Comb. 9, 75 (2005); J. Differ. Equations Appl. 11, 799 (2005); Ann. Comb. (2009) to appear, arXiv:0808.2596 Proceedings of the Conference on Motives, Quantum Field Theory, and Pseudodifferential Operators, to appear in the Mathematics Clay Proceedings, (2010); Sém. Lothar. Combin. 56, 1 (2007), Article B56b, Habilitationsschrift JKU Linz (2007) and references therein.

139. J. Blümlein, M. Kauers, S. Klein and C. Schneider, Comput. Phys. Commun. 180, $2143(2009)$.

140. J. A. M. Vermaseren New features of FORM, (2000), math-ph/0010025

141. P. Cartier (2002), Sém. Bourbaki, Mars 2001, 53e année, exp. no. 885, Asterisque 282 137-173.

142. V. V. Zudilin, Uspekhi Mat. Nauk 56, 149 (2001).

143. S. L. Adler, Phys. Rev. 177, 2426 (1969).

144. J. S. Bell and R. Jackiw, Nuovo Cim. A60, 47 (1969).

145. C. Bouchiat, J. Iliopoulos and P. Meyer, Phys. Lett. B38, 519 (1972). 